\title{
Vestibular evoked myogenic potential testing
}

\section{Payment policy review for clinicians and payers}

Terry D. Fife, MD, Saty Satya-Murti, MD, Robert F. Burkard, MD and John P. Carey, MD

Neurology: Clinical Practice April 2018 vol. 8 no. 2 129-134 doi:10.1212/CPJ.0000000000000430

Correspondence

Dr. Fife

tfife@email.arizona.edu

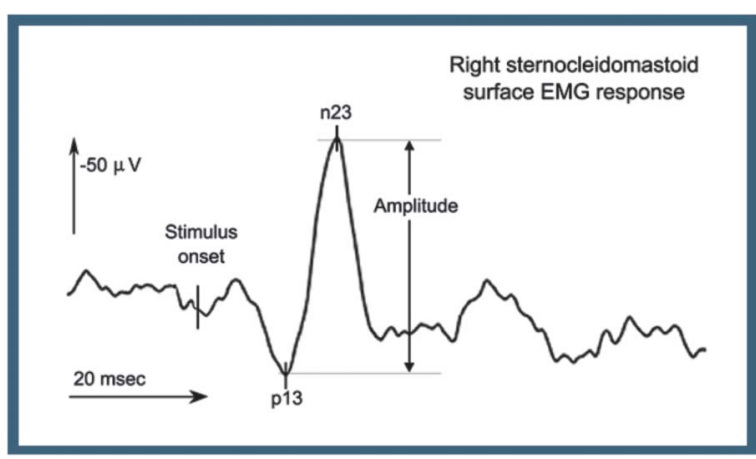

test and also explores payment policies for VEMP.

\section{Recent findings}

In carefully selected patients with documented history compatible with the SCDS, both highresolution temporal bone CT scan and VEMP are valuable aids for diagnosis. Payers might be unfamiliar with both this syndrome and VEMP testing.

\section{Summary}

It is important to raise awareness of VEMP and its possible indications and the rationale for coverage of VEMP testing. Payers may not be readily receptive to VEMP coverage if this test is used in an undifferentiated manner for all common vestibular and auditory symptoms.

VEMP is a relatively new vestibular function test performed by stimulating one ear with repetitive pulse or click sound stimulation and then measuring surface EMG responses over selected muscles averaging the reaction of the muscle electrical activity associated with each sound click or pulse. The clinical application of VEMP was first reported by Colebach in 1994 but had its basis in even earlier work in the vestibular field. ${ }^{1}$ While VEMP testing has been increasingly used throughout the world, its use in the United States has been more limited. Recently, the American Academy of Neurology published an evidence-based practice guideline on the use of VEMP. In addition, in October 2015 the FDA approved VEMP equipment by GN Otometrics (Schaumburg, IL) for sale in the United States. In March 2017, Interacoustics (Middelfart, Denmark) and in September 2017 Intelligent Hearing Systems (Miami, FL) were also granted FDA approval to market VEMP equipment sales. This article is intended to help clinicians incorporate the conclusions of the recently published practice guideline ${ }^{2}$ and to offer special attention to payment policy issues for this new diagnostic test.

Department of Neurology (TDF), Barrow Neurological Institute and University of Arizona College of Medicine, Phoenix; Health Policy Consultant (SS-M), Santa Maria, CA; Department of Rehabilitation Science (RFB), University of Buffalo, NY; and Department of Otolaryngology-Head and Neck Surgery (IPC), Johns Hopkins School of Medicine, Baltimore, MD. 
VEMP serves as a physiologic confirmation in those suspected of

\section{having SCDS and is a useful adjunct to the temporal bone CT.}

\section{Major guideline findings}

The guideline related to VEMP use in the diagnosis of SCDS concluded that clinicians may use cVEMP stimulus threshold values to distinguish SCDS from controls and that corrected cVEMP amplitude may be used to distinguish SCDS from controls. In addition, clinicians may use oVEMP amplitude to distinguish SCDS from normal controls and oVEMP threshold may be used to aid in distinguishing SCDS from controls. Table 1 outlines sensitivity and specificity data from the guideline. It was also concluded that there is currently insufficient evidence to determine whether cVEMP and oVEMP can accurately identify vestibular function specifically related to the saccule/utricle, or whether cVEMP or oVEMP is useful in diagnosing vestibular neuritis or Ménière disease (Level C).

There are 2 types of VEMP testing: cVEMP and oVEMP. A sound stimulus applied to one ear evokes a response within the ipsilateral sternocleidomastoid muscles that can be recorded with surface electrodes and averaged (cVEMP) (figure 1A). Similarly, a sound stimulus to the ear also evokes a response that is largest when recorded from the contralateral inferior oblique muscle recorded by a surface electrode and averaged (oVEMP) (figure 1B).

VEMP serves as a physiologic confirmation in those suspected of having SCDS and is a useful adjunct to the temporal bone CT.

\section{Superior canal dehiscence syndrome}

SCDS is an uncommon syndrome due to an abnormal opening or dehiscence in the bony roof of the superior semicircular canal in the temporal bone. ${ }^{4}$ In contrast to other more well-recognized diagnoses capable of causing vertigo,
SCDS is an unfamiliar entity to many. This unfamiliarity leads to under-recognition of the entity. This condition may present with excessive sound sensitivity, sometimes by pulsatile tinnitus as well as a variety of sensations of dizziness including dizziness evoked or triggered by changes in sound or pressure. ${ }^{5}$

\section{Suspecting SCDS}

There are no established diagnostic criteria for SCDS but clinical suspicion should be piqued by a report of dizziness induced by coughing, sneezing, straining, or loud sounds. Hearing one's own bone-conducted sounds such as pulse, footsteps, or speech is another typical feature (autophony). Dizziness caused by sounds, vibration, and altitude changes along with sound sensitivity can be suggestive of SCDS and prompt workup with thin cut temporal bone imaging looking for the appearance of dehiscence. High-resolution CT of the temporal bone is typically needed for the diagnosis and is essential before consideration of surgical intervention.

False-positives have been reported with temporal bone CT studies. ${ }^{6,7}$ CT may overcall the anatomic finding of dehiscence because of a number of technical factors. ${ }^{8}$ Moreover, an opening in the bone over the superior canal (superior canal dehiscence [SCD]) may not actually transmit pressure until the dura becomes sufficiently elastic (e.g., from aging or trauma). Thus, VEMP testing may aid in the diagnosis by serving as a physiologic correlate to the anatomic appearance on temporal bone CT. By combining temporal bone CT with VEMP, it appears very likely that the diagnostic accuracy for SCDS will improve. ${ }^{5}$ In properly diagnosed instances of SCDS, surgical repair of the dehiscence carries a high rate of successful relief of symptoms ${ }^{9}$ and normalization of VEMP abnormalities. ${ }^{10}$

Without clear documentation, by history and examination, the rationale for suspecting the presence of SCDS and the legitimacy for testing become tenuous.

A discriminating pretest clinical evaluation for symptom severity is critical in promoting shared decision-making.

Table 1 Sensitivity and specificity findings of the practice guideline on vestibular evoked myogenic potential (VEMP) testing for superior canal dehiscence syndrome (SCDS) ${ }^{2}$

\begin{tabular}{|c|c|c|c|}
\hline Test measure for diagnosis of SCDS & Sensitivity, ${ }^{a} \%$ & Specificity, ${ }^{a} \%$ & Level of recommendation \\
\hline Cervical VEMP threshold $<2$ SD below that of controls ${ }^{c}$ & $86-91$ & $90-96$ & Possibly predictive of SCDS (Level C) ${ }^{\mathrm{b}}$ \\
\hline $\begin{array}{l}\text { Corrected cervical VEMP amplitude }>2 \text { SD greater than } \\
\text { that of controls }\end{array}$ & 100 & 93 & Possibly predictive of SCDS (Level C) \\
\hline Ocular VEMP threshold $<2$ SD below that of controls & $77-100$ & $77-100$ & Possibly predictive of SCDS (Level C) \\
\hline Ocular VEMP amplitude $>2$ SD greater than controls & $77-100$ & $98-100$ & Possibly predictive of SCDS (Level C) \\
\hline
\end{tabular}

${ }^{\text {a }}$ Values of sensitivity and specificity are for distinguishing those with SCDS from healthy controls.

${ }^{\mathrm{b}}$ Level of recommendation as described in Edlund et al. ${ }^{3}$

${ }^{c}$ Normative values based on control patients should be established in each individual laboratory. 
Figure 1 Waveform for cervical vestibular evoked myogenic potential (VEMP) and for ocular VEMP
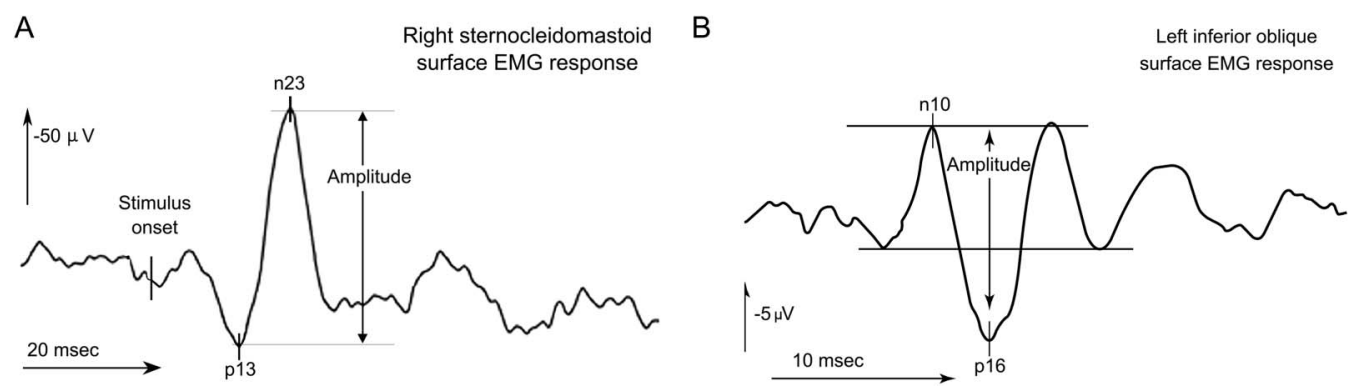

(A) Cervical VEMP. (B) Ocular VEMP.

Patients who choose to have surgery have more severe presurgical autophony, auditory, or vestibular symptoms. ${ }^{11}$

\section{Diagnosing SCDS}

As of now, other than the clinical evaluation, there is no established reference test (gold standard) against which a putative diagnostic procedure for SCDS can be evaluated. In diagnostic medicine, the availability of an incomplete or absent reference standard is not an unfamiliar situation. Generally a diagnostic test, even if less than perfect, by virtue of its earlier appearance and longevity becomes the reference standard. In the absence of a perfect reference standard, some alternatives may serve in lieu of a rigid gold standard for making decisions. They are as follows: results of several tests, an "umpire" test, natural history of the disease process, and clinical follow-up and prognosis after treatment. ${ }^{12-15}$ In SCDS, surgery can confirm the anatomic finding of dehiscence. Resolution of symptoms along with normalization of physiologic abnormalities after repair also confirms a role of VEMPs in the diagnosis of SCDS. ${ }^{10}$ Until remaining uncertainties clear, newer diagnostic technologies deserve periodic evaluations rather than outright acceptance or rejection.

\section{Diagnostic algorithm options}

There is no established algorithm for the best way to diagnose and confirm SCDS. Figures 2 and 3 show 2 reasonable algorithms for diagnosing clinically suspected instances of SCD based on current evidence. The first suggestion is to perform high-resolution CT scans of the temporal bone followed by a confirmatory VEMP, especially when surgery is planned. The second is to carry out VEMP testing as a screening test with a subsequent CT scan if the VEMP is anything other than normal, especially when surgery is being considered. One of these diagnostic pathways or perhaps another will be clarified with more clinical experience and over time.

\section{Arguments in support of performing VEMP}

The above sequence for suspecting, differentiating, and diagnosing SCDS is valuable in seeking insurance coverage either prior to a procedure or building an argument against denial of payment after a test has been performed. It is important for clinicians to ensure a solid prior probability before ordering VEMP. This sets a convincing backdrop to show that VEMP is not used by the provider as a routine indiscriminate test in persons with the rather common clinical complaints of dizziness, tinnitus, hearing difficulties, or imbalance. Such nonspecific use of VEMPs will dilute the value of the test, and subsequently solidify payers' notions that these tests are of low health benefit.

However, clinicians who evaluate patients for vertigo or dizziness know that it is rare that one test clinches the diagnosis (e.g., Dix-Hallpike testing for benign paroxysmal positional vertigo $[\mathrm{BPPV}])$. Instead, in the majority of cases, diagnosis arises from the recognition of syndromic patterns from the history, examination, and quantitative testing. For example, a patient with superior vestibular neuritis has about a $30 \%$ chance of developing posterior canal BPPV subsequently, ${ }^{16}$ which is believed to be due to release of otoconia from inflammation that became located in the posterior canal to cause BPPV. Here an intact cVEMP is expected since the inferior vestibular nerve supplies the posterior canal and the saccule is still functional and could cause subsequent BPPV. Such a case illustrates that cVEMP may have important diagnostic and management implications, even though it is not diagnostic in isolation.

\section{Advantages of VEMP}

VEMP is a noninvasive safe test that is similar to other established evoked potential tests already in use. Its helpfulness in clarifying the correct diagnosis can result in improved outcomes. Surgery for SCDS, based on large case series, indicates a good level of success in alleviating symptoms with very low failure rates when properly diagnosed. Its

\section{VEMP is a noninvasive safe test that is}

\section{similar to other established evoked} potential tests already in use. 
Figure 2 One reasonable algorithm option for using vestibular evoked myogenic potential (VEMP) in the diagnosis of superior canal dehiscence syndrome (SCDS)

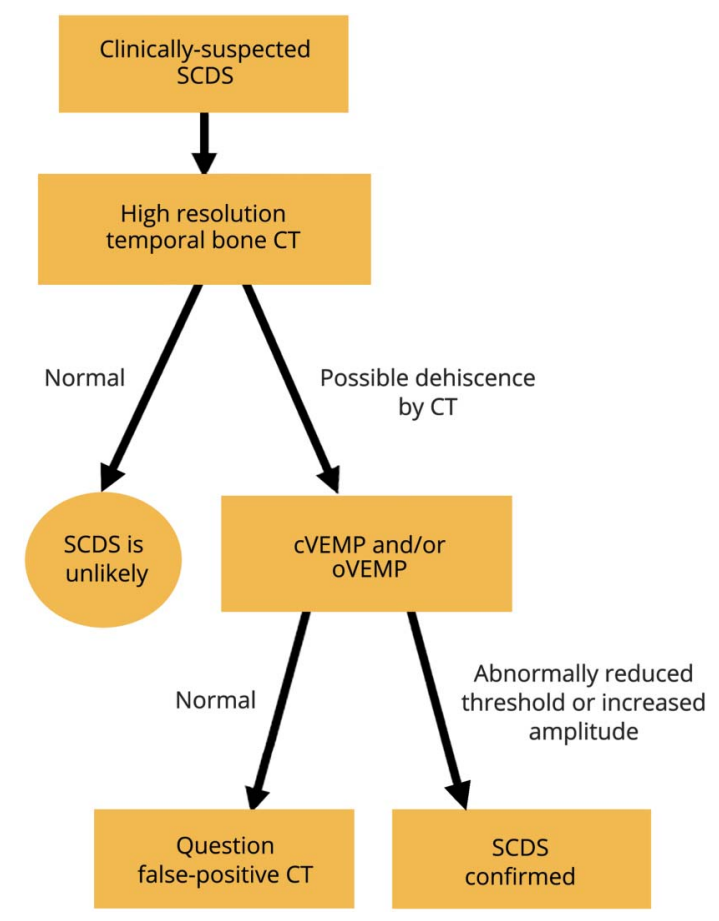

cVEMP = cervical VEMP; oVEMP = ocular VEMP

utilization is unlikely to be high when utilized narrowly to aid in the diagnosis of SCDS.

\section{Disadvantages or limitations of VEMP}

The lack of a perfect diagnostic reference standard becomes a persuasive argument for mindful selection of patients for VEMP testing. In view of the very satisfactory rate of symptom resolution with surgical repair, attention to historical details and confirmation through one of the diagnostic pathways is necessary prior to surgery. Awareness of the potential for indiscriminate testing for nonspecific auditoryvestibular symptoms is essential. It is not unreasonable to expect payer scrutiny due to overutilization of testing modalities. This advice is especially applicable to unfamiliar, underrecognized symptom complexes and newer test modalities.

VEMP is an evoked potential test like auditory brainstem responses (ABRs) though with less history, less familiarity in clinical use, and no history of insurance coverage in the United States. The technical and interpretive work of VEMP is almost identical to ABRs.

There are no dedicated procedure codes assigned to cVEMP or oVEMP. The comparability to BAEP is likely to ease comprehension of concepts by payers. In the meantime, patients should be issued a procedure-specific Advanced
Figure 3 A second reasonable algorithm option for use of vestibular evoked myogenic potential (VEMP) in making the diagnosis of superior canal dehiscence syndrome (SCDS)

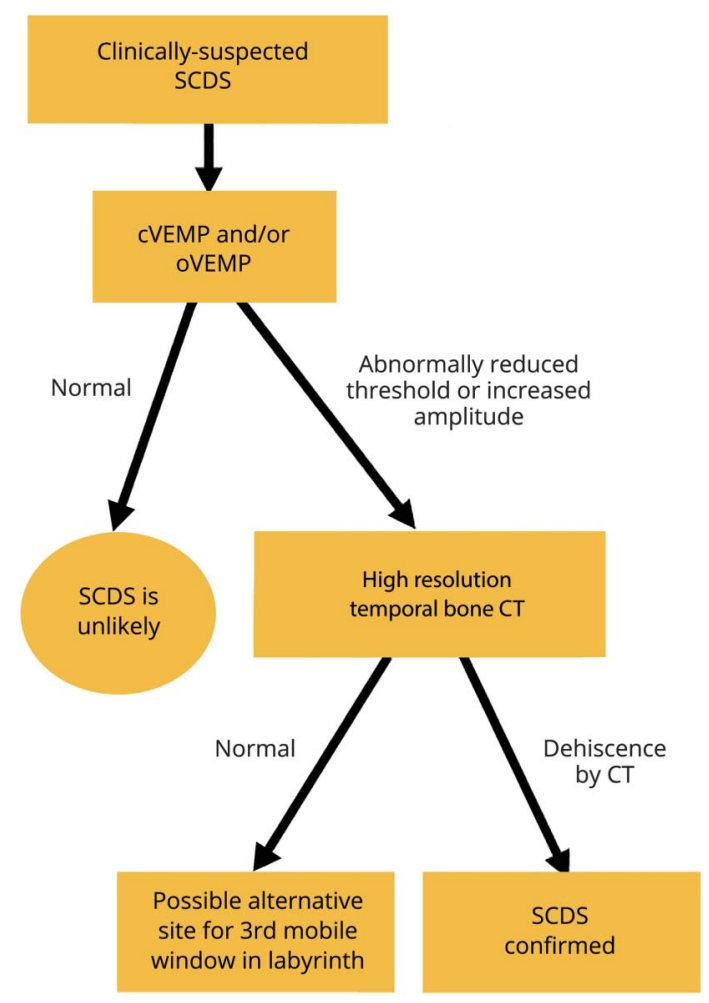

cVEMP = cervical VEMP; oVEMP = ocular VEMP.

Beneficiary Notice of noncoverage to clarify that VEMP procedures are not usually paid for by Centers for Medicare $\&$ Medicaid Services (CMS) and by some other payers.

\section{Current coverage policies}

Medicare contractors may elect to question the need for VEMPs not only because of unfamiliarity but also due to the findings of variable accuracy in older persons. Consequently, private payers should not wait for CMS or Medicare administrative contractors to issue coverage determinations but rather develop their own coverage policies based on the current literature.

Many insurance companies require prior authorization on unlisted procedures in order for payment to be considered. Several insurance companies have issued payment policies related to VEMP. Aetna payment policy, last reviewed on April 4, 2017, cites older reviews of vestibular testing from $2000^{17}$ and $2006^{18}$ indicating this modality is still investigational. ${ }^{19}$ Blue Cross Blue Shield of Rhode Island policy, last updated on April 18,2017 , concludes that evidence is insufficient to determine the effects of VEMP on health outcomes. ${ }^{20}$ Healthnet has a national medical policy, last updated in July 2016, and does not cover VEMP due to a lack of evidence from well-controlled prospective clinical trials showing VEMP alters management or 
Table 2 Suggestions for engaging in a payer dialogue when vestibular evoked myogenic potential (VEMP) test value comes under review

1. Anticipate the need to explain to reviewers and payers both the entity of superior canal dehiscence syndrome and the nature of VEMPs.

2. Use appropriate sections of the guideline, and this article, for additional support.

3. Explain how your group/practice selects patients based on carefully elicited history characteristic of the syndrome. Be willing to produce appropriate documentation.

4. Be prepared for discussion of situations where reference gold standards may not be available.

5. Stress the comparability of VEMP work (technical and interpretive) to more familiar evoked potential tests such as brainstem auditory evoked potential testing.

6. Be aware of the absence of specific procedure codes for VEMP.

improves outcome. ${ }^{21}$ Paramount medical policy on vestibular function testing from January 10, 2017, states that the US FDA has not approved specific devices for VEMP testing (though, as mentioned earlier, the FDA has now reversed its stance and has approved devices from 2 separate companies). The policy concludes that VEMP testing is investigational and therefore not a covered procedure. ${ }^{22}$

\section{Conclusions}

VEMP testing and SCDS may be unfamiliar to many clinicians and payers. The recently published practice guideline may use VEMP testing as a way of separating patients with SCDS from controls, thus aiding in making the diagnosis of SCDS. Additional research is needed to clarify how to best use VEMP in arriving at a diagnosis of SCDS in clinical practice. Figures 2 and 3 depict 2 reasonable algorithms for use of VEMP in diagnosing SCDS. Table 1 summarizes sensitivity and specificity data from the practice guideline and table 2 offers some suggestions in preparation for discussion with payers on the utility of VEMP testing for this diagnosis. At present, there is insufficient evidentiary support for use of VEMP in general dizziness and so we would not anticipate this to be heavily utilized save for the narrow indication of suspected SCDS unless new evidence emerges. Due to lack of CPT code, one can anticipate coding as a miscellaneous code would undergo focused medical review by nongovernmental insurers. Clinicians must recognize and be prepared to argue for the value of VEMPs in circumstances other than SCDS when the test may refine diagnosis and guide management. An appreciation for VEMPs in a holistic evaluation of vestibular function, incorporating history, neuro-otologic examination, and appropriate auditory and other quantitative vestibular testing, is essential in arguing what is best for clinical care.

\section{Author contributions}

T.D. Fife: contributed to the concept and drafting of the article and revising it critically for important intellectual content and approves the submitted version of this article. S. Satya-Murti: contributed to the concept and drafting of the article and revising

\section{TAKE-HOME POINTS}

$\rightarrow$ Vestibular evoked myogenic potential (VEMP) testing is a relatively new vestibular testing technique that determines vestibular function by applying a repetitive sound stimulus to one ear and then averaging the reaction of the muscle activity in response to each sound click or pulse.

$\rightarrow$ Unlike caloric testing or rotational chair testing, VEMP does not measure eye movements. Surface EMG recordings over the ipsilateral sternocleidomastoid muscle (cervical VEMP [CVEMP]) is believed to assess function of the saccule. Surface EMG recordings over the contralateral inferior oblique (ocular VEMP [OVEMP]) is believed to assess function of the utricle.

$\rightarrow$ In a comprehensive evidence-based review of the literature, weak evidence supports VEMP testing as valuable in affirming the diagnosis of superior canal dehiscence syndrome (SCDS) but so far, quality evidence is limited for its routine use in other vestibular disorders.

$\rightarrow$ As a new test just approved by the Food and Drug Administration (FDA) about 2 years ago and currently without a specific billing procedure code (i.e., Current Procedural Terminology [CPT] code), it is anticipated that some payers will require clarification or explanation before approving payment until payment policies are updated and a specific CPT code is assigned.

it critically for important intellectual content and approves the submitted version of this article. R.F. Burkard: contributed to revising the article critically for important intellectual content and approves the submitted version of this article. J.P. Carey: contributed to revising the article critically for important intellectual content and approves the submitted version of this article. 


\section{Study funding}

No targeted funding reported.

\section{Disclosure}

T.D. Fife serves on 2 data safety and monitoring boards for NIH-sponsored studies. S. Satya-Murti has participated in telephone consultations or in-person medical advisory board meetings for Evidera (formerly United BioSource Corporation UBC), Simon-Kucher consultants, Baxter, CB Partners, Avalere LLC, Medtronic, 1798 Consultants, Parexel, DeciBio, and AbbVie; has received funding for travel from United BioSource Corporation UBC (2010-13), AstraZeneca, Avalere LLC, Evidera consulting group, Covidien, Michael J. Fox Foundation, Foley Hoag LLP, Baxter, and AbbVie; serves on the editorial board of Neurology ${ }^{\oplus}$ : Clinical Practice; served on the American Academy of Neurology Payment Policy Subcommittee; and served as panelist and later (2010-2011) Vice-Chair CMS-MEDCAC (Medicare Evidence Development and Coverage Advisory Committee). For the duration of the MEDCAC meeting, usually 1-2 days, S. Satya Murti was considered a Special Government Employee (SGE). R.F. Burkard serves on an Advisory Board for the NCRAR at the Portland VA Hospital; is a member of the Board of Directors for the American Auditory Society and a member of the Health Care Economics Committee for the American Speech-Language-Hearing Association; and has served as a paid consultant on a grant given to the Marine Mammal Foundation. J.P. Carey serves on a DSMB for NIH-funded clinical trial; has received funding for travel from Otonomy; serves as an Associate Editor for Audiology and Neurotology; has served as a consultant for Pfizer and Otonomy; and performs vestibular testing interpretation $(<10 \%$ effort; VEMPs are a fraction of that); if recommendations were fully implemented, they would actually reduce VEMP revenue; and receives research support from Otonomy and NIH/NIDCD. Full disclosure form information provided by the authors is available with the full text of this article at Neurology.org/cp.
Received September 4, 2017. Accepted in final form November 28, 2017.

\section{References}

1. Zhou G, Cox LC. Vestibular evoked myogenic potentials: history and overview. Am J Audiol 2004; 13:135-143.

2. Fife TD, Colebatch JG, Kerber KA, et al. Practice guideline: cervical and ocular vestibular evoked myogenic potential testing. Neurology 2017;89:2288-2296.

3. Edlund W, Gronseth G, So Y, Franklin G. Clinical practice guideline process manual. Minneapolis: American Academy of Neurology; 2004.

4. Minor LB, Solomon D, Zinreich JS, Zee DS. Sound- and/or pressure-induced vertigo due to bone dehiscence of the superior semicircular canal. Arch Otolaryngol Head Neck Surg 1998; 124:249-258.

5. Ward BK, Carey JP, Minor LB. Superior canal dehiscence syndrome: lessons from the first 20 years. Front Neurol 2017;8:177.

6. Mikulec AA, McKenna MJ, Ramsey MJ, et al. Superior semicircular canal dehiscence presenting as conductive hearing loss without vertigo. Otol Neurotol 2004;25:121-129.

7. Masaki Y. The prevalence of superior canal dehiscence syndrome as assessed by temporal bone computerized tomography imaging. Acta Otolaryngol 2011;131:258-262.

8. Tavassolie TS, Penninger RT, Zuñiga MG, Minor LB, Carey JP. Multislice computed tomography in the diagnosis of superior canal dehiscence: how much error, and how to minimize it? Otol Neurotol 2012;33:215-222.

9. Shaia WT, Diaz RC. Evolution in surgical management of superior canal dehiscence syndrome. Curr Opin Otolaryngol Head Neck Surg 2013;21:497-502.

10. Welgampola MS, Myrie OA, Minor LB, Carey JP. Vestibular-evoked myogenic potential thresholds normalize on plugging superior canal dehiscence. Neurology 2008; 70:464-472.

11. Alkhafaji MS, Varma S, Pross SE, Sharon JD, et al. Long-term patient-reported outcomes after surgery for superior canal dehiscence syndrome. Otol Neurotol 2017;38: 1319-1326.

12. Rutjes AW, Reitsma JB, Coomarasamy A, et al. Evaluation of diagnostic tests when there is no gold standard. A Review Methods 2007;11:iii, ix-51.

13. Glasziou P, Irwig L, Deeks JJ. When should a new test become the current reference standard? Ann Intern Med 2008;149:816-822.

14. Gold R, Reichman M, Greenberg E, et al. Developing a new reference standard: is validation necessary? Acad Radiol 2010;17:1079-1082.

15. Statistical Guidance on Reporting Results from Studies Evaluating Diagnostic Tests. 2007. FDA. Available at: fda.gov/medicaldevices/deviceregulationandguidance/ guidancedocuments/ucm071148.htm. Accessed August 25, 2017.

16. Murofushi T, Halmagyi GM, Yavor RA, Colebatch JG. Absent vestibular evoked myogenic potentials in vestibular neurolabyrinthitis: an indicator of inferior vestibular nerve involvement? Arch Otolaryngol Head Neck Surg 1996;122:845-848.

17. Fife TD, Tusa RJ, Furman JM, et al. Assessment: vestibular testing techniques in adults and children: report of the Therapeutics and Technology Assessment Subcommittee of the American Academy of Neurology. Neurology 2000;55:1431-1441.

18. Rauch SD. Vestibular evoked myogenic potentials. Curr Opin Otolaryngol Head Neck Surg 2006;14:299-304.

19. Aetna Policy 2017. Evoked potential studies, number 0181, last reviewed $4 / 4 / 17$, next review 2/8/18. Available at: aetna.com/cpb/medical/data/100_199/0181.html. Accessed April 10, 2017.

20. Blue Cross Blue Shield (of Rhode Island) Policy 2017. Medical coverage policy on vestibular function tests, last updated 4/18/17. Available at: bcbsri.com/sites/default/ files/polices/Vestibular\%20Function\%20Tests.pdf. Accessed August 10, 2017.

21. Healthnet Policy 2016. Healthnet national medical policy: vestibular function testing. 2016. Available at: healthnet.com/portal/provider/content/iwc/provider/unprotected/ working_with_HN/content/medical_policies.action\#V. Accessed May 10, 2017.

22. Paramount Policy 2017. Paramount medical policy on vestibular function testing, policy PG0323, last reviewed $1 / 10 / 17$. Available at: paramounthealthcare.com/ documents/MedicalPolicy/PG0323_Vestibular_Function_Testing.pdf. Accessed April 10, 2017. 